\title{
Validity of the new nutrition screening tool Control of Food Intake, Protein, and Anthropometry (CIPA) in non-surgical inpatients
}

\author{
José Pablo Suárez-Llanos ${ }^{1}$, Alejandra Mora-Mendoza ${ }^{1}$, Néstor Benítez-Brito ${ }^{1}$, Lina Pérez-Méndez², \\ Francisca Pereyra-García-Castro ${ }^{1}$, José Gregorio Oliva-García ${ }^{1}$, José Enrique Palacio-Abizanda ${ }^{1}$
}

\begin{abstract}
${ }^{1}$ Endocrinology and Nutrition Department, Hospital Universitario Nuestra Señora de Candelaria, Santa Cruz de Tenerife, Spain

${ }^{2}$ Research Unit, Hospital Universitario Nuestra Señora de Candelaria and Primary Healthcare, CIBER CIBER, Respiratory Diseases, Instituto de Salud Carlos III, Madrid, Spain
\end{abstract}

Submitted: 17 May 2016

Accepted: 18 September 2016

Arch Med Sci 2018; 14, 5: 1020-1024

DOI: https://doi.org/10.5114/aoms.2017.66084

Copyright (c) 2017 Termedia \& Banach

\begin{abstract}
Introduction: There is no gold-standard method for hospital nutrition screening. The new screening tool termed Control of Food Intake, Protein, and Anthropometry (CIPA) gives positive results when at least one of the following parameters is met: control of food intake for $72 \mathrm{~h}<50 \%$, serum albumin $<3 \mathrm{~g} / \mathrm{dl}$, body mass index $<18.5 \mathrm{~kg} / \mathrm{m}^{2}$ or mid-upper arm circumference $\leq 22.5 \mathrm{~cm}$. This method was validated in comparison with Subjective Global Assessment (SGA) in hospitalized patients with non-surgical pathologies.

Material and methods: A prospective, longitudinal study was performed on 221 consecutively enrolled patients. Prevalence or risk of malnutrition was estimated with CIPA vs. SGA screening at hospital admission and the concordance $(\kappa$ index $-K$ ) between the two methods and their sensitivity $(S)$ and specificity (SP) were studied. Mean length of stay (LOS), mortality, and rate of early readmission were analyzed.

Results: The prevalence or risk of malnutrition identified by CIPA and SGA was $35.7 \%$ and $23.1 \%$, respectively. $K$ was 0.401 ( $p<0.001)$; S and SP of CIPA vs. SGA were $72.5 \%$ and $75.3 \%$, respectively. In contrast to SGA, CIPA-positive patients had an increased mean LOS compared to the negative ones (19.53 vs. 12.63 days, $p<0.001)$. Both methods detected a major risk of mortality in positive patients, but no difference in early readmission.

Conclusions: The CIPA and the SGA screening tools detect patients with a higher risk of mortality, but only CIPA identifies patients with an increased mean LOS. CIPA screening proved valid for use in non-surgical inpatients.
\end{abstract}

Key words: malnutrition, screening, health care quality, inpatient, nutrition assessment.

\section{Introduction}

Hospital malnutrition (HM), usually disease-associated, frequently occurs not only in developing but also developed countries. Depending on the population studied and the employed detection method, its prevalence ranges from $25 \%$ to $50 \%$, as confirmed in a number of multicenter studies, such as ELAN (in Latin America, with over 9000 pa-

\author{
Corresponding author: \\ José Pablo Suárez-Llanos \\ Endocrinology and \\ Nutrition Department \\ Hospital Universitario \\ Nuestra Señora \\ de Candelaria \\ 145 Carretera del Rosario St \\ 38010 Santa Cruz de Tenerife \\ Spain \\ Phone: +34678754003 \\ E-mail: pablua@hotmail.com
}


tients enrolled), EuroOOPS (European, over 5000 patients), and PREDYCES (Spanish, over 1700 patients) [1-4].

The association between malnutrition and the occurrence of comorbidities in the form of increased risk of infections, fistulas, delayed healing and recovery of the patient is well known and leads to poor functional capacity, increased length of stay (LOS), higher health care costs, and increased mortality $[3,5,6]$. These data contrast with the scarce attention paid to HM and shortage of hospitals in Europe that incorporate nutritional screening programs [4]. In addition, it has been recently observed that malnourished patients who are treated with nutritional support improve in the above-mentioned clinical as well as economic parameters $[7,8]$.

There is no internationally established gold standard hospital nutrition screening, so that the most adequate would be the most feasible within the characteristics of each hospital. This type of screening should predict patient outcome, be inexpensive without being time-consuming, and most caregivers should be able to perform it [9].

The Control of Food Intake, Protein, and Anthropometry (CIPA) nutrition screening tool was designed at the Hospital Universitario Nuestra Señora de Candelaria (HUNSC, Tenerife, Spain), taking into account routinely assessed parameters, i.e. 72-h food intake control, serum albumin, and body mass index (BMI) or mid-upper arm circumference (MUAC) in patients who cannot be weighed or their height measured [10-12]. Since then, this screening method has been employed at hospital admission and thereafter, when negative, every 10 days to detect patients malnourished during hospitalization. It was set up in almost all the hospital wards in 2015, regardless of the challenge of installing a nutrition screening method in a hospital setting [13].

In light of the lack of a reference hospital nutrition screening method, Subjective Global Assessment (SGA) is recommended for comparison and evaluation of the efficacy of any nutrition screening method and was therefore applied as such [14, 15].

\section{Material and methods}

\section{Subjects}

A prospective study was performed on $221 \mathrm{pa}$ tients of both sexes, hospitalized in the different wards of the HUNSC hospital in the period from January to June 2014. The study was approved by the ethics committee of the hospital. All patients provided written informed consent for their participation.

Exclusion criteria were the admission of patients to surgical wards, an admission for foreseeably less than three days, nutritional support prior to the third day of hospitalization - as prolonged screening does not make sense in patients receiving specific nutritional support - and a lack of informed consent. Subjects were recruited from the patients consecutively admitted to the wards where the CIPA screening was implemented (Pulmonology, Internal Medicine, Gastroenterology, Nephrology, and Oncology).

\section{Measures}

The following baseline data were collected: age, sex, admitting ward, weight $(\mathrm{kg})$, height $(\mathrm{m})$, and BMI $\left(\mathrm{kg} / \mathrm{m}^{2}\right)$ or MUAC $(\mathrm{cm})$. The same doctor performed both CIPA and SGA screenings in all patients at $72 \mathrm{~h}$.

The CIPA outcomes were considered positive if one of the following conditions occurred: (1) 72-h food intake control with intakes below $50 \%$, (2) serum albumin $<3 \mathrm{~g} / \mathrm{dl}$, (3) BMI $<18.5 \mathrm{~kg} / \mathrm{m}^{2}$ or MUAC $\leq 22.5 \mathrm{~cm}$ (in subjects where body weight or height could not be determined). In the event of a positive CIPA outcome, the patient received nutritional support if the responsible doctor considered the measure appropriate. Although CIPA was performed repeatedly every for 10 days when outcomes were negative, the present work only reflects the screening data from the moment of admission.

Hence, patients were considered malnourished or at risk when at least one of the CIPA screening parameters was positive or SGA screening resulted in categories B or C.

In order to clinically validate the CIPA screening tool, the following clinical parameters were analyzed in terms of positivity for either of the two screening systems: LOS (days), early readmission (< 1 month; \%), and mortality in hospital as well as including the first month after discharge (\%).

\section{Statistical analysis}

Data are expressed as percentage, mean \pm standard deviation (SD), or median with interquartile range (IQR) depending on the distribution of variables. Differences between categorical variables were compared using Pearson's $\chi^{2}$ test or Fisher's exact test. For continuous variables, data were analyzed using Student's $t$-test.

In order to validate the applicability of CIPA scoring to detect $\mathrm{HM}, 95 \%$ confidence intervals were calculated to estimate sensitivity and specificity. Inter-rater agreement was assessed using the $\kappa$ coefficient. Data were analyzed using SPSS for Windows, version 21.0. A two-tailed $p$-value $<0.05$ was considered statistically significant.

\section{Results}

The baseline features of the 221 consecutively enrolled study patients are listed in Table I. 
Table I. Baseline characteristics of the sample

\begin{tabular}{|c|c|c|}
\hline Parameter & Mean & SD \\
\hline Age [years] & 64 & 13.9 \\
\hline Weight [kg] & 75.6 & 17.28 \\
\hline BMI $\left[\mathrm{kg} / \mathrm{m}^{2}\right]$ & 28.21 & 6.4 \\
\hline Parameter & $n$ & $\%$ \\
\hline Total & 221 & 100 \\
\hline \multicolumn{3}{|l|}{ Gender: } \\
\hline Male & 128 & 57.9 \\
\hline Female & 93 & 42.1 \\
\hline \multicolumn{3}{|l|}{ Services: } \\
\hline Pneumology & 95 & 43 \\
\hline Internal Medicine & 46 & 20.8 \\
\hline Digestive & 53 & 24 \\
\hline Nephrology & 17 & 7.7 \\
\hline Oncology & 10 & 4.5 \\
\hline
\end{tabular}

$S D$ - standard deviation, $B M I$ - body mass index.

The prevalence or risk of suffering malnutrition was $35.7 \%$ (95\% Cl: 29.2-42.3), determined by CIPA, and $23.1 \%$ (95\% Cl: 6.0-15.0), determined by SGA screening. Patients with positive CIPA had an increased LOS compared to CIPA negative ones (19.53 days, $95 \% \mathrm{Cl}: 14.0-25.0$, vs. 12.63 days, 95\% Cl: 11.0-14.2, $p<0.001)$, which contrasted with SGA screening (17.39 days, 95\% Cl: 13.121.7 , vs. 14.4 days, $95 \% \mathrm{Cl}: 12.4-16.4, p=0.162$ ) (Figure 1). Positive screening by either method detected patients at increased risk of death in the course of hospitalization (CIPA: $7.6 \%$ vs. $1.4 \%, p=$ 0.026 ; SGA: $9.8 \%$ vs. $1.8 \%, p=0.017$ ) (Figure 2 ) as well as including the first month after discharge (positive CIPA: $12.7 \%$ vs. $3.5 \%, p=0.012$; positive SGA: $15.7 \%$ vs. $4.1 \%, p=0.002$ ) (Figure 3 ). Rates of early readmission did not differ significantly between positive screening outcomes with either method.

The degree of concordance between CIPA and SGA screening ( $\kappa$ index $-K)$ was $0.401(95 \% \mathrm{Cl}$ : $0.27-5.03, p=0.001)$. Taking SGA as a reference, the sensitivity and specificity of CIPA screening were $72.5 \%$ and $75.3 \%$, respectively.

\section{Discussion}

Despite the deleterious effect of malnutrition in hospitalized patients, only a few hospitals perform nutrition screening at admission. In addition to the scarce sensitivity to this phenomenon among managers, politicians, and health care professionals in general, the lack of a reference screening

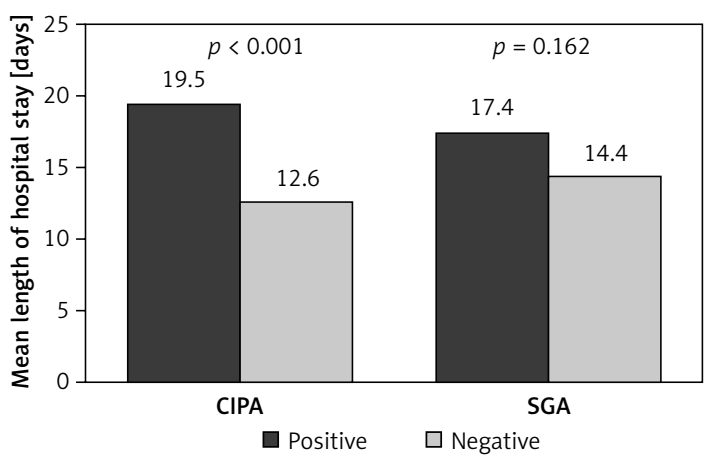

Figure 1. Length of hospital stay assessed by CIPA vs. SGA screening tool

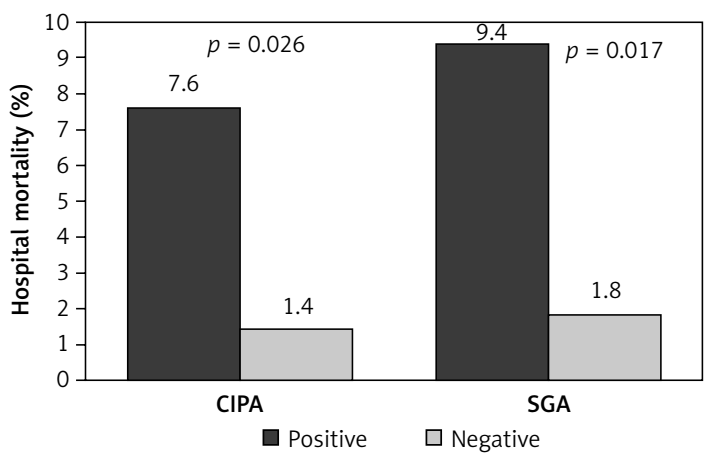

Figure 2. In-hospital mortality assessed by CIPA vs. SGA

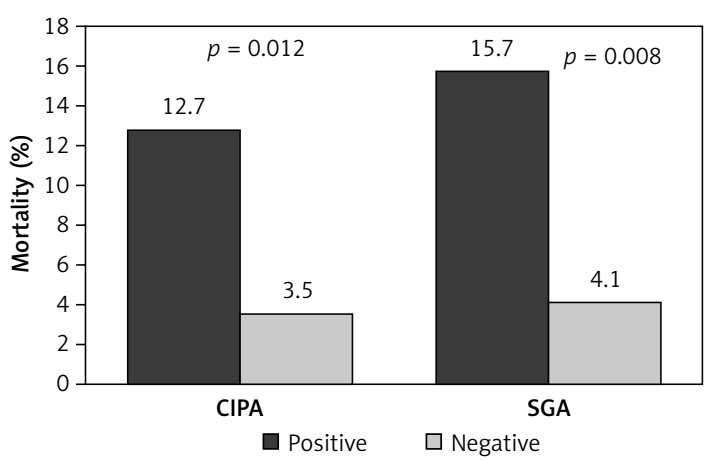

Figure 3. In-hospital mortality including the first month after discharge assessed by CIPA vs. SGA screening tool

method contributes to a failure of universal implementation.

Nutrition screening tools which better predict the clinical course of the patient, such as NRS2002 [16] or SGA [17], are difficult to perform in hospitals with many beds, as they are complex and time-consuming to execute, while simpler ones, such as SNAQ [18] or MST [19], do not predict the patient's course equally well. Alternatively, the Mini-Nutritional Assessment (MNA), in its short version, complies with all the requisites but is intended for elderly patients and cannot be fully introduced in a non-geriatric hospital [20].

Therefore, our working group has designed the CIPA screening tool, which comprises central as- 
pects of nutrition assessment - such as the analysis of food intake, protein and anthropometric parameters - and exploits activities routinely performed in our hospital. In addition, this method encompasses essential features, such as simplicity, no need for skilled health care personnel, and low costs, which only the little time spent by the health staff and for serum albumin sampling account for.

A positive aspect of the CIPA screening method is its objectivity, as the only parameters with a certain degree of subjectivity comprise the analysis of intake controls by the nursing staff and the identification of patients who, on average, consume less than half of the meal served at the hospital. However, the concordance between the interpretations of the nursing personnel and a nutritionist (the reference personnel in this study) was very good $(K=0.8, p<0.001)$ [12].

The good reproducibility is important as it implies that any health care personnel can carry out the screening procedure. By contrast, SGA requires qualified personnel to perform the physical examination. As this entails a subjective component, the results may vary with the interpretation and capacity of the observer.

Another helpful feature of the CIPA screening tool is that it does not require patients' cooperation. Hence, they need not answer any question - otherwise problematic in patients with cognitive impairment or memory disorders - nor do they have to get up for anthropometric measurement, when not feasible, as the MUAC measurement would apply in such a case. The latter constitutes an advantage over other types of screening. It should be kept in mind that patient malnutrition is usually associated with a reduced functional capacity, and that the phenomenon emerges more frequently in elderly patients and patients with cognitive impairment, where the need to collaborate in nutrition screening would be a handicap.

This work shows that the patients who tested positive on CIPA screening had a significantly longer hospital stay, i.e. for one additional week. The mean LOS is the ideal parameter to evaluate associated complications and consequently the increase in health costs $[5,21]$. In our patient sample, CIPA screening was more effective than SGA in this regard; the latter did not detect any difference.

Another variable that can gage the clinical course of a patient is the rate of early readmission, but neither method was capable of identifying patients at increased risk. However, both methods detected patients with a higher mortality rate, both in hospital and during the month after discharge. Although SGA detected higher mortality in terms of numbers, the mortality risk was similar with both screening methods, so that patients who tested positive on either screen- ing had a more than five times increased risk of in-hospital mortality than patients with a negative outcome and an almost four times higher risk including the first month after discharge.

A nutrition screening tool should not only detect malnourished patients - whose defining parameters are not even entirely clear at present [22] - but must identify patients with a higher rate of complications than the rest [9]. The CIPA screening tool met this criterion in the studied sample, so that patients with a worse prognosis were detected by assessing the included nutrition parameters.

There was a significantly higher prevalence or risk of suffering malnutrition on CIPA than on SGA screening. This could imply either that CIPA provides numerous false positives or that certain patients are underdiagnosed through SGA. In the present study, SGA provided low sensitivity compared with the CIPA screening tool as to the clinical prognoses in our patient sample. Therefore, the fact that the degree of concordance between the two methods was not good does not detract from the value of the CIPA screening tool. Consistent with these data, the authors of a recently published meta-analysis concluded that SGA does not seem to work better than many other screening methods [23].

Also, considering SGA as the reference method, the sensitivity and specificity of CIPA screening surpass what an efficient screening tool is meant to accomplish (> 70\%) [24].

As for the limitations of this study, it should be mentioned that patients were enrolled consecutively, when admitted to the hospital, and were subsequently subjected to nutrition screening. Therefore, the patient sample was not homogeneous in terms of hospital wards.

In conclusion, the CIPA screening method was validated in in-hospital patients admitted for nonsurgical pathologies and was found to meet the requirements of an appropriate nutrition screening tool, i.e. simplicity, practicability, low costs, and the capability to predict the clinical course of patients, including mortality.

\section{Conflict of interest}

The authors declare no conflict of interest.

\section{References}

1. Edington J, Boorman J, Durrant ER, et al. Prevalence of malnutrition on admission to four hospitals in England. The Malnutrition Prevalence Group. Clin Nutr 2000; 19: 191-5.

2. Alvarez-Hernandez J, Planas-Vila M, Leon-Sanz M, et al. Prevalence and costs of malnutrition in hospitalized patients: the PREDyCES Study. Nutr Hosp 2012; 27: 1049-59.

3. Sorensen J, Kondrup J, Prokopowicz J, et al. EuroOOPS: an international, multicentre study to implement nutri- 
tional risk screening and evaluate clinical outcome. Clin Nutr 2008; 27: 340-9.

4. Correia MI, Campos AC. Prevalence of hospital malnutrition in Latin America: the multicenter ELAN study. Nutrition 2003; 19: 823-5.

5. Correia MI, Waitzberg DL. The impact of malnutrition on morbidity, mortality, length of hospital stay and costs evaluated through a multivariate model analysis. Clin Nutr 2003; 22: 235-9.

6. Golabek T, Powroźnik J, Chłosta P, Dobruch J, Borówka A. The impact of nutrition in urogenital cancers. Arch Med Sci 2015; 11: 411-8.

7. Elia M, Normand C, Norman K, Laviano A. A systematic review of the cost and cost effectiveness of using standard oral nutritional supplements in the hospital setting. Clin Nutr 2016; 35: 370-80.

8. Philipson TJ, Snider JT, Lakdawalla DN, Stryckman B, Goldman DP. Impact of oral nutritional supplementation on hospital outcomes. Am J Manag Care 2013; 19: 121-8.

9. Waitzberg DL, Correia MI. Nutritional assessment in the hospitalized patient. Curr Opin Clin Nutr Metab Care 2003; 6: 531-8.

10. Suárez Llanos JP, Benitez Brito N, Oliva García JG, et al. Introducing a mixed nutritional screening tool (CIPA) in a tertiary hospital. Nutr Hosp 2014; 29: 1149-53.

11. Benítez Brito N, Suárez Llanos JP, Fuentes Ferrer $M$, et al. Relationship between mid-upper arm circumference and body mass index in Inpatients. PLoS One 2016; 11: e0160480.

12. Benítez Brito N, Mora Mendoza A, Suárez Llanos JP et al. Concordance in the results of control intake performance of $72 \mathrm{~h}$ by different health professionals in a tertiary hospital. Nutr Hosp 2015; 32: 2895-9.

13. Burden ST, Brierley ER. Evaluation of adherence to a nutrition screening programme over a 5 -year period. Eur J Clin Nutr 2014; 68: 847-52.

14. Van Bokhorst-de van der Schueren MA, Guaitoli PR, Jansma EP, de Vet HC. Nutrition screening tools: does one size fit all? A systematic review of screening tools for the hospital setting. Clin Nutr 2014; 33: 39-58.

15. Velasco C, García E, Rodriguez V, et al. Comparison of four nutricional screening Tools to detect nutricional risk in hospitalizad patients: a multicentre study. Eur J Clin Nutr 2011; 65: 269-74.

16. Kondrup J, Rasmussen HH, Hamberg O, Stanga Z. Nutritional risk screening (NRS 2002): a new method based on an analysis of controlled clinical trials. Clin Nutr 2003; 22: 321-36.

17. Detsky AS, McLaughlin JR, Baker JP, et al. What is subjective global assessment of nutritional status? JPEN J Parenter Enteral Nutr 1987; 11: 8-13.

18. Kruizenga HM, Seidell JC, de Vet HC, Wierdsma NJ, van Bokhorst-de van der Schueren MA. Development and validation of a hospital screening tool for malnutrition: the short nutritional assessment questionnaire (SNAQ). Clin Nutr 2005; 24: 75-82.

19. Ferguson M, Capra S, Bauer J, Banks M. Development of a valid and reliable malnutrition screening tool for adult acute hospital patients. Nutrition 1999; 15: 458-64.

20. Rubenstein LZ, Harker JO, Salva A, Guigoz Y, Vellas B. Screening for undernutrition in geriatric practice: developing the short-form mini-nutritional assessment (MNA-SF). J Gerontol A Biol Sci Med Sci 2001; 56: M366-72.

21. Lobo Támer G, Ruiz López MD, Pérez de la Cruz AJ. Hospital malnutrition: relation between the hospital length of stay and the rate of early readmissions. Med Clin 2009; 132: 377-84.

22. Cederholm T, Bosaeus I, Barazzoni R, et al. Diagnostic criteria for malnutrition - an ESPEN Consensus Statement. Clin Nutr 2015; 34: 335-40.

23. da Silva, FJ, Mello P, De Luis D, Mello E. Subjective global assessment of nutritional status - a systematic review of the literature. Clin Nutr 2015; 34: 785-92.

24. Neelemat F, Meijers J, Kruizenga $H$, Van Ballegooijen $H$, van Bockhorst-de van der Schueren. Comparison of five malnutrition screening tools in one hospital inpatient sample. J Clin Nurs 2011; 20: 2144-52. 